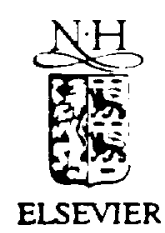

Optical Materials 1387 (1996) xxx C

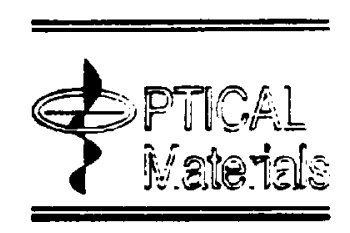

NASA-CR-201106

\title{
Effect of variations in the doping profiles on the properties of doped multiple quantum well avalanche photodiodes
}

\author{
H.M. Menkara, B.K. Wagner, C.J. Summers \\ Quantum Microstructure Branch, Georgia Tech Research Institure Atlanta, Ga 30332-086I. USA
}

Accepted 17 January 1996

\begin{abstract}
The purpose of this study is to use both theoretical and experimental evidence to determine the impact of doping imbalance and symmetry on the physical and electrical characteristics of doped multiple quantum well avalanche photodiodes. Theoretical models have been developed to calculate the electric field, valence and conduction bands. capacitance-voltage $(C V)$, and carrier concentration versus depletion depth profiles. The models showed a strong correlation between the $p$ - and $n$-doping balance inside the GaAs wells and the number of depleted stages and breakdowin voltage of the APD. A periodic loping imbalance in the wells has been shown to result in a gradual increase (or decrease) in the electric field profile throughout the device which gave rise to parially depleted devices at low bias. The MQW APD structures that we modeled consisted of a $1 \mu \mathrm{m}$ top $\mathrm{p}^{+}-$doped $\left(3 \times 10^{18} \mathrm{~cm}^{-3}\right) \mathrm{GaAs}$ layer, followed by a $1 \mu \mathrm{m}$ region of alternating layers of $\operatorname{GaAs}(500 \AA)$ and $\mathrm{Al}_{0.42} \mathrm{Ga}_{038} \mathrm{As}(500 \AA)$, and a $1 \mu \mathrm{m} \mathrm{n} \mathrm{n}^{+}$back layer $\left(3 \times 10^{18} \mathrm{~cm}^{-3}\right)$. The GaAs wells were doped with $p^{-j}-n$ layers placed at the center of each well. The simulation results showed that in an APD with nine doped wells. and where the $50 \AA$ p-doped layer is off by $10 \%\left(p=1.65 \times 10^{18} \mathrm{~cm}^{-3} \cdot n=1.5 \times 10^{18} \mathrm{~cm}^{-3}\right)$, almost half of the MQW' stages were show'n to be undepleted at low bias which was a result of a reduction in the electric field near the $\mathrm{p}^{+}$ cap layer by over $50 \%$ from its value in the balanced structure. Experimental $C V$ and $N$ data on similar MBE grown MQW structures have shown very similar depletion and breakdown characteristics. The models have enabled us to better interpret our experimental data and to determine both the extent of the doping imbalances in The devices as well as the overall p-or n-type doping characteristics of the structures.
\end{abstract}

\section{Introduction}

Extensive studies have been performed on multiple quantum well (MQW) structures because of their potential applications in avalanche photodiodes (APDs) and tunneling devices [1]. The MQW structure was first proposed by Chin et al. [2] and $\mathrm{Ca}$ passo et al. [3] as a method to enhance the electronhole ionization ratio $(k=\alpha / \beta)$ beyond that in bulk materials. Later, Blauvelt [4] proposed the doped MQW structure to further increase $k$. The desire for high performance optical detectors has resulted in several proposed MQW APD structures using different material systems in an attempt to optimize their gain, noise, and bandwidth characteristics.

Various characterization techniques had been devised to analyze and understand the optical and electrical properties of APDs [5]. These include such experiments as current-voltage (IV) measurements which are used to determine the gain properties of the device, and capacitance-voltage ( $C V$ ) measurements which are used to calculate carrier concentra- 
tion versus depletion width profiles. However, the data obtained using such experimental techniques are nol always easy to interpret and relate back to the physical processes taking place inside the structures. In addition, the practical limitations inherent into the growth and fabrication of large quantities of devices with different structural designs add even more complexity to the problem because of the presence of multiple degrees of freedom.

In this paper, we present a more practical approach to analyzing and expanding our knowledge of the experimental data obtained using $N$ and $C V$ experiments and, specifically, those relating the doping profile to device properties. We have developed accurate theoretical models of MQW APD structures using Atlas II, Silvaco's two-dimensional device simulation framework. The models were used to provide graphical representations of the spatial variations of the electric field across the biased structure, as well as conduction and valence band diagrams of the GaAs/AlGaAs MQW structure before and after breakdow'n. In addition, avalanche breakdown simulations and small signal ac analysis were used to extract $N$ and $C V$ curves in order to compare the data from the models to those obtained directly from the experimental devices. Both electron- and hole-injected photocurrent solutions were obtained by simulating a $632.8 \mathrm{~nm}$ monochromatic light source of about $1 \mathrm{~W} / \mathrm{cm}^{2}$ incident on the devices' front and back surfaces. $\mathrm{CV}$ solutions were obtained using small signal analysis at a frequency of $1 \mathrm{MHz}$ and with a signal magnitude of $0.03 \mathrm{~V}$. The analysis was conducted using Newton's two-carrier method [6] to solve the carrier continuity and Poisson's equations. The generation rate of electron-hole pairs due to impact ionization was modeled according to Selberherr [7].

\section{Theoretical analysis}

The APD structure used in the modelling consisted of a top and bottom $\mathrm{p}^{+}$and $\mathrm{n}^{+}$doped $\left(3 \times 10^{18}\right.$ $\mathrm{cm}^{-3}$ ) GaAs layers with a thickness of $1 \mu \mathrm{m}$ and an area of $75 \mu \mathrm{m}^{2}$. The middle region was made up of 10 periods of alternating layers of GaAs $(500 \AA)$ and

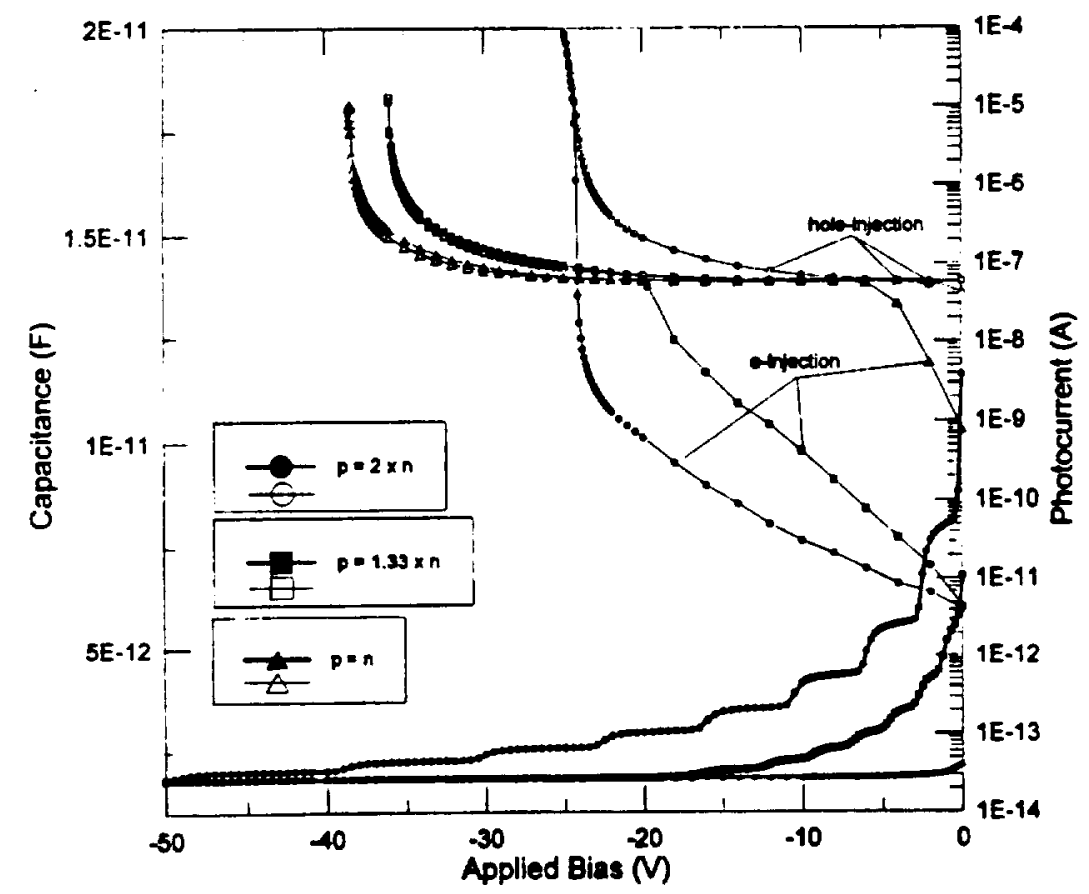

Fig. 1. Comparison of theoretical $C V$ and light $N$ curves obtained for the same APD MQW structure where the doping mismatch in the wells was varied between $0 \%$ and $100 \%$. 
$A I_{0.42} \mathrm{Ga}_{0.58}$ As $(500 \AA)$. The GaAs wells were similarly doped with $p-i-n$ layers where the $p$ - and $n$-doping concentrations were treated as variable parameters for the purpose of this study. When a reverse bias is applied, the combined effect of the applied electric field, the built-in field, and the conduction band offset enhances the ionization process of electrons in the GaAs. The holes, on the other hand, are subjected to a smaller valence band discontinuity and therefore gain less energy than their electrons counterparts.

For this study, the widths of the $p$ and $n$ doping layers in the wells were beld constant at $50 \AA$, and that of the intrinsic layer at $100 \AA$. The doping imbalance $(|p|-|n|) /|n|$ was varied between zero and $200 \%$. This was done by holding the $n$-doping constant at $1.5 \times 10^{18} \mathrm{~cm}^{-3}$ and increasing the $\mathrm{p}$ doping from $1.5 \times 10^{18} \mathrm{~cm}^{-3}$ to $4.5 \times 10^{18} \mathrm{~cm}^{-3}$. Fig. I shows theoretical $C V$ and reverse light $N$ plots (for both electron and hole injection) obtained for similar devices with $0 \%, 33.3 \%$, and $100 \%$ dop- ing imbalances. As expected, the capacitance of the device is lowest when the $p$ - and $n$-doping are perfectly matched since the net carrier concentration throughout the device is reduced to zero. In addition, the avalanche breakdown voltage as depicted by the $I V$ curves, seems to decrease whenever the doping in the wells is unbalanced. This is due to the fact that a doping mismatch would result in a gradual increase of the electric field throughout the device which would cause impact ionization to take place at a lower bias point (see Fig. 2). Therefore, a large doping imbalance would actually help lower the bias at which breakdown occurs as shown by the $I V$ plots in Fig. 1.

Fig. 3 shows the carrier concentration versus depletion width profile for similar APD structures with $0 \%, 10 \%, 33.3 \%, 100 \%$, and $200 \%$ doping mismatches. In the case where $p=n=1.5 \times 10^{18} \mathrm{~cm}^{-3}$. we can see the device is fully depleted at zero bias. As the imbalance between the $\mathrm{p}$ - and $\mathrm{n}$-doping is increased, the number of undepleted stages increases.

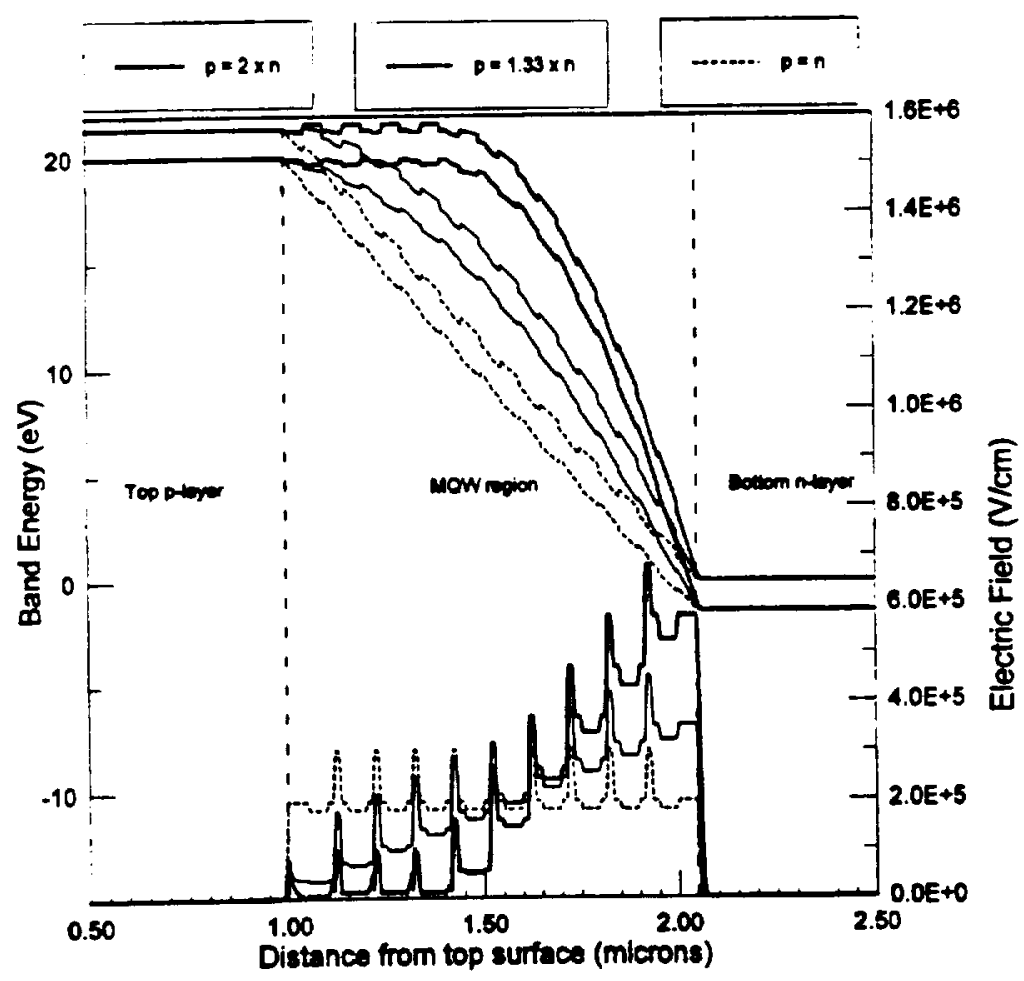

Fig. 2. Theoretical band diagrams and electric field profiles of the same MQW APD structures with different doping imbalance in the wells. 
In the case where the p-doping is twice that of $n$, we find that only about $20 \%$ of the device's MQW region is depleted at zero bias. Reducing the doping imbalance to as low as $10 \%$ would only increase the depletion layer width to $50 \%$ of the device's MQW region, thereby requiring additional bias to reach full depletion.

In order to better understand the effect of the doping imbalance on the MQW structures, we will examine the valence and conduction band diagrams,

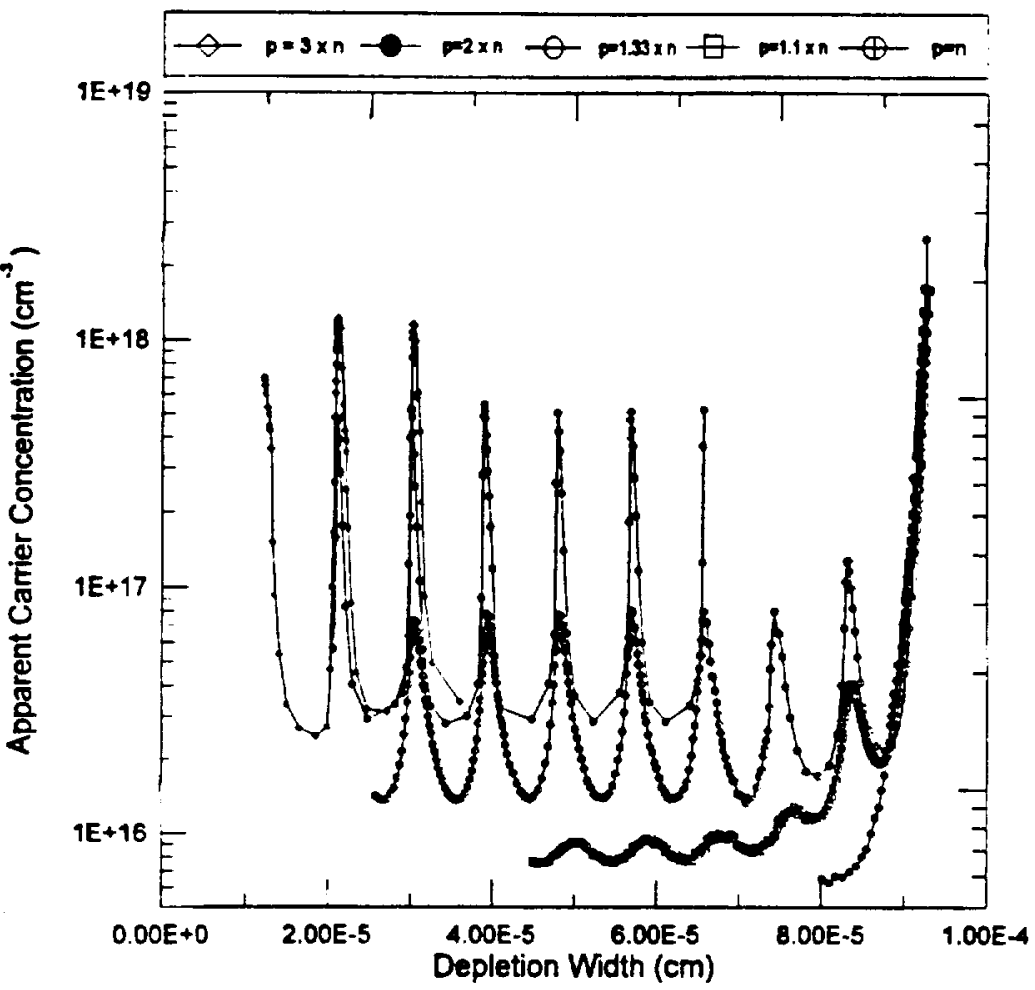

Fig. 3. Calculated doping profiles versus depletion width using theoretical $C V$ data obtained from ac analysis on similar MQW APDs with different doping imbalance in wells.

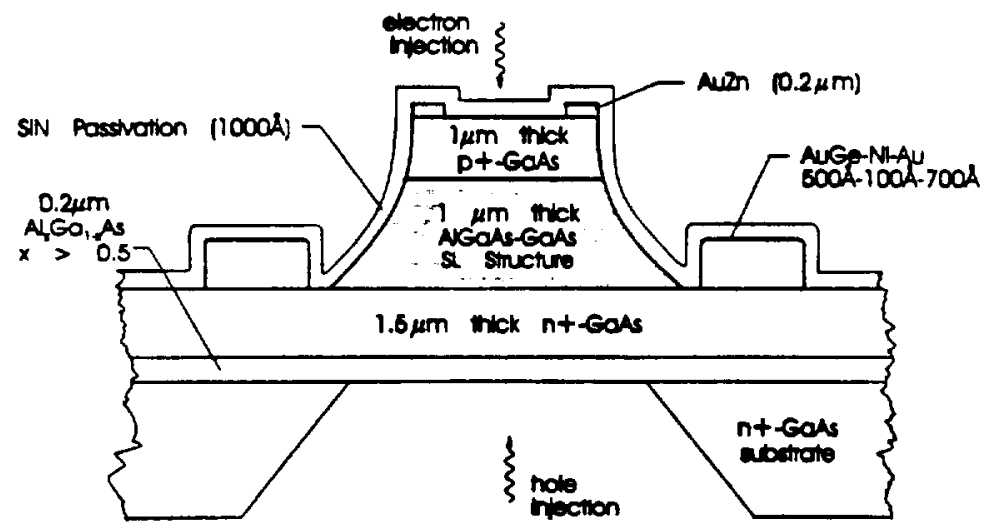

Fig. 4. Cross section of a 10-period MQW APD 
as well as the electric field profile shown in Fig. 2. We ve selected the three devices corresponding to $0 \%, 33 \%$, and $100 \%$ doping imbalances being operated at a reverse bias of $20 \mathrm{~V}$. As we can see from the top and bottom diagrams, in the case where $p=n$, the electric field is uniformly symmetric and the band diagram equally depleted. However, as $p$ gradually increases, the electric field becomes progressively lower near the top p-layer which results in non-uniform depletion of the MQW structure. The effect of such non-uniform depletion on the device's photocurrent can be clearly seen in the $I V$ plots shown in Fig. 1. In the case of electron injection, the photocurrent at zero bias is about four orders of magnitude lower than that for hole injection. This is because the undepleted AlGaAs regions near the p-layer present a high energy barrier to the injected electrons and limit the photocurrent. Gradually, however, the electron-injected photocurrent increases as the device is depleted until it reaches the level corresponding to that of the hole-injected photocurrent (see Fig. 1). Therefore, a doping imbalance where $p>n$ can greatly reduce the device's external quantum efficiency in the case of electron injection. Such an effect is not as pronounced in the case where $n>p$ due to the smaller valence band discontinuity faced by the injected holes.

\section{Experimental results}

All of our APD devices were grown by molecular beam epitaxy (MBE) in a Varian Gen $I$ system and were fabricated using standard photolithographic techniques. Fig. 4 shows a cross section of an experimental MQW APD where contact rings were used to provide a uniform electric field across the entire device. The MQW region in all devices consisted of 10 sets of alternating layers of GaAs $(500 \AA)$ and $\mathrm{Al}_{0.42} \mathrm{Ga}_{0.58}$ As $(500 \AA)$ with $1000 \AA$ periods. All

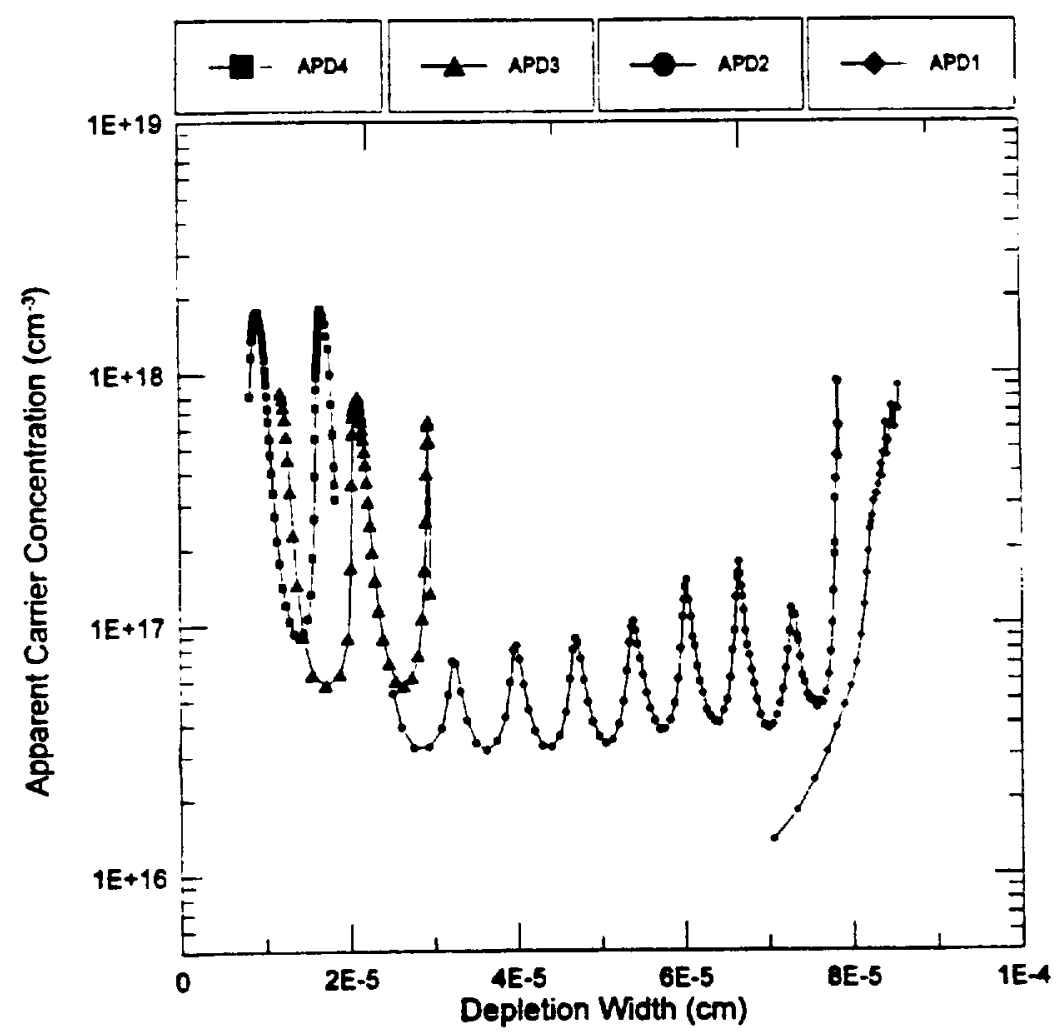

Fig. 5. Calculated doping profile versus depletion width using experimental CV data from four 10-period doped-well MQW APDs. 
APDs were composed of a $1 \mu \mathrm{m}$ Be-doped $\left(3 \times 10^{18}\right.$ $\left.\mathrm{cm}^{-3}\right) \mathrm{p}^{+}$top layer, and a $1.5 \mu \mathrm{m}$ Si-doped $\left(3 \times 10^{18}\right.$ $\left.\mathrm{cm}^{-3}\right) \mathrm{n}^{+}$back layer. In the MQW devices, high electric fields were achieved in the narrow bandgap GaAs wells of the avalanche region through the introduction of thin $(50-150 \AA)$ and highly doped $\left(0.5 \times 10^{18}-1.5 \times 10^{18} \mathrm{~cm}^{-3}\right) \mathrm{p}^{+}$and $\mathrm{n}^{+}$layers. Electron injection was achieved by focusing a 632.8 $\mathrm{nm}$ HeNe laser inside the circular p-contact ring. $C V$ measurements were performed on all devices at 1 MHz using an HP4277A LCZ meter. The $C V$ data was then analyzed to calculate the depletion widths and carrier profiles for the structures. Fig. 5 shows the net carrier concentration plots for all four doped-well MQW devices labeled as APDI through APD4. All devices have similar geometries except for the "intended" $p$ - and n-doping in the wells which varied between $0.5 \times 10^{18}$ to $1.5 \times 10^{18} \mathrm{~cm}^{-3}$. lt is interesting to see that even thoughall APDs were expected to have very similar properties, their $\mathrm{CV}$ and carrier concentration profiles were quite different. APD1 was almost fully depleted at zero bias, while APD2 was not and only reached full depletion right before breakdown. Both APD3 and APD4 were only partially depleted even at the onset of avalanche which explains their low breakdown voltage.

Note that most of the peak positions in the carrier profile plots do not quite agree with the $1000 \AA$ period in the MQW structure and with the doping profiles obtained using the models. This discrepancy is due to the fact that carria concentration profiles were calculated assuming a one sided depletion [8] which apparently does not hold toue for the actual devices. Other sources of error in the experimental data result from the inability to accurately account for parasitic capacitance between the devices and the metal contacts and bonding wires in the measurement system. In addition, note how the average net

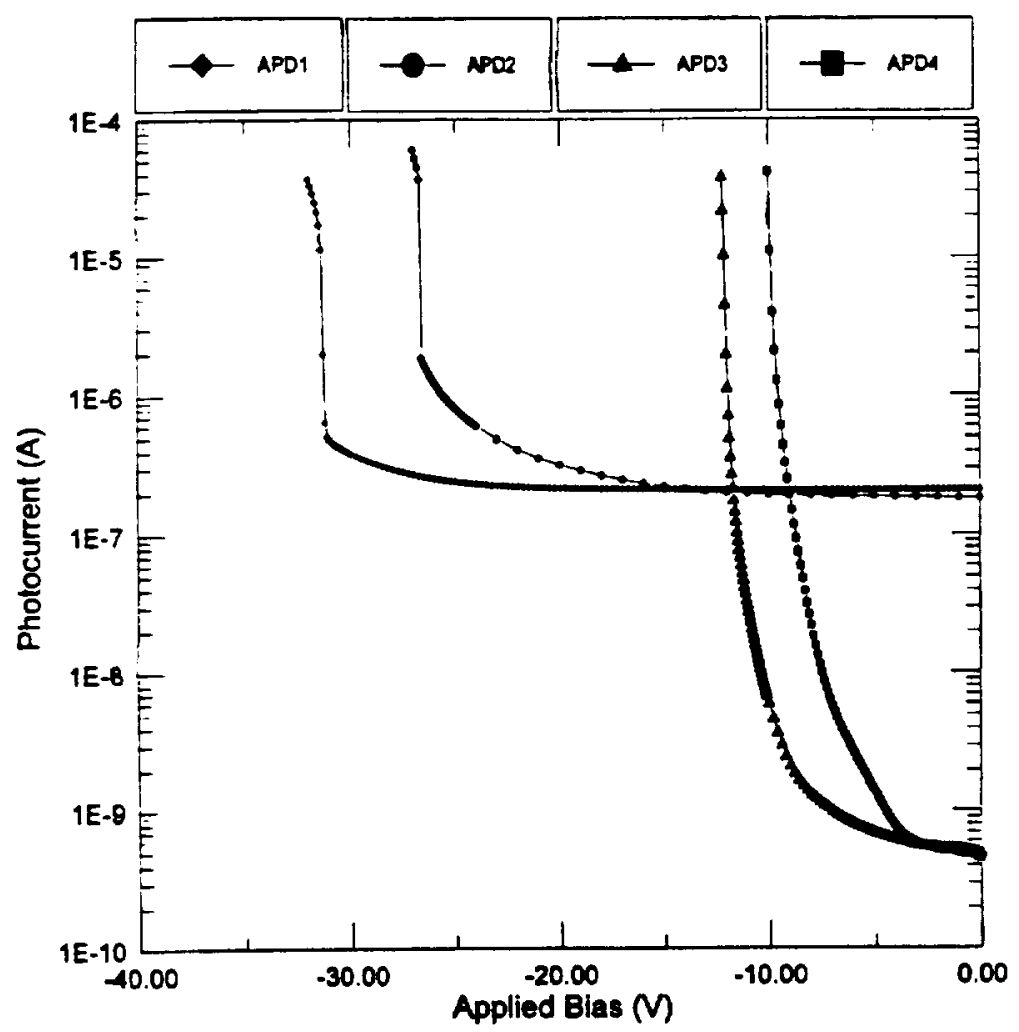

Fig. 6. Experimental light $N$ curves obtained for all four 10-period doped-well MQW APDs for the same incident photon flux (electron injection). 
carrier concentration in the experimental doping profile of APD2 is gradually increasing up until the top GaAs well where it drops indicating that the doping imbalance is not the same throughout the structure.

The spatial resolution of the $C-V$ measurements (both experimental and theoretical) was limited by the Debye length which is about $40 \AA$ at room temperature for a doping level of $n=1.5 \times 10^{18}$ $\mathrm{cm}^{-3}$. Since the thickness of the doped layers in the wells was of the same order of magnitude ( $50 \AA$ ), abrupt changes in the doping concentration could not be accurately measured. Therefore, it is generally difficult to relate the apparent carrier concentration obtained from the experimental devices to the actual doping imbalance in the wells. However, using the theoretical carrier profile data shown in Fig. 3 where the actual doping imbalance is fully known, it is possible to estimate the actual doping mismatch in every doped layer in the experimental MQW device by superimposing both the experimental and theoretical data. By examining Figs. 3 and 5 , we can roughly conclude that the average doping imbalance in APD 1 is far less than $10 \%$ resulting in full depletion at zero bias, while that in APD2 is between $30 \%$ and $40 \%$ where full depletion of the MQWs was achieved near breakdown around $27 \mathrm{~V}$. In the case of APD3 and APD4, the situation was quite different. Apparently, the $\mathrm{p}$ - and $\mathrm{n}$-doping mismatch was so large $(-200 \%)$ to the point where only partial depletion of two wells took place before avalanche breakdown. By examining the electron injected photocurrent curves shown in Fig. 6, we can conclude that for both APD1 and APD2, the doping mismatch is such that $n>p$, while in APD3 and APD4, the situation is reversed. This can be clearly understood by comparing the light $I V$ data in Fig. 6 to the theoretical curves shown in Fig. 1. The gradual increase in the electron injected photocurrent in APD3 and APD4 is an indication of trapping of injected electron by the AIGaAs barriers near the p-layer where the device is undepleted. Therefore, according to the models shown in Fig. 2, the average p-doping in the wells must be larger than that of $n$. In the case of APD1 and APD2, no trapping seems to take place since the low bias photocurrent is much higher and relatively flat. Therefore, these two devices have undepleted regions near bottom $n$-layer which indicates that $n>p$.

\section{Conclusions}

We have made a full investigation of the impact of doping imbalance in doped-well MQW APDs on the devices' $I V$ and $C V$ characteristics and how such an imbalance would affect the depletion properties of the APDs. Our theoretical models were in full agreement with the observed experimental data and have helped us develop a good understanding of some of the physical processes that take place inside a doped MQW APD. Our simplistic models have enabled us to easily interpret experimental $I V$ and $C V$ data and determine the extent of depletion in the APD devices and how all of these quantities are affected by the pand $n$-doping imbalance in the structure. We have found that a doping mismatch as small as $10 \%$ can reduce the depletion layer width by as much as $50 \%$. We've also found that a large doping imbalance would cause the device to quickly reach avalanche breakdown in the depleted layers and would prevent full depletion. Finally, using electron-injected photocurrent $N$ data, we were able to determine wether the overall doping imbalance throughout the structure is positive $(p>n)$ or negative $(n>p)$ and the extent of the imbalance was determined by comparing both the experimental and theoretical carrier concentration profiles.

\section{Acknowledgements}

This work was sponsored by NASA under contract number NAGW-2753. Travel expenses were provided in part by the Microelectronics Research Center (MiRC) at the Georgia Institute of Technology.

\section{References}

[1] F. Capasso, Semiconductors and Semimetals. R.K. Willardson and A.C. Beer, eds. (Academic Press. New York, 1985), Vol. 22, par D. p. 2.

[2] R. Chin, N. Holoniak, G.E. Stillman, J.Y. Tsang and K. Hess, Appl. Phys. Lett. 16 (1980) 467.

[3] F. Capasso, W.T. Tsang, A.L. Hutchinson and P.G. Williams. Appl. Phys. Let1. 40 (1982) 38.

[4] H. Blauvelt, S. Margalit and A. Yariv, Electron. Lett. 18 (1982) 375 . 
(5) H.M. Menkara. B.K. Wagner and C.J. Summers, Appl. Phys. Lell. 66 (1995) 1764.

(6) Silvaco Intemational. Atlas Il User's Manual, 1st ed. (1993).

17] S. Selberherr. Analysis and Simulation of Semiconductor De- vices (Springer, Vienna, 1984).

[8] N. Yamamato, K. Yokoyama and M. Yamamato. Appl. Phys. Len. 62 (1993) 252. 